\title{
Pathology of serrated colorectal lesions
}

\author{
Adrian C Bateman
}

Correspondence to Dr Adrian C Bateman, Department of Cellular Pathology, University Hospital Southampton NHS Foundation Trust, MP002, Level E, South Block, Southampton General Hospital, Tremona Road, Southampton S016 6YD, UK; adrian.bateman@uhs.nhs.uk

Received 8 January 2014 Accepted 14 January 2014 Published Online First 21 February 2014
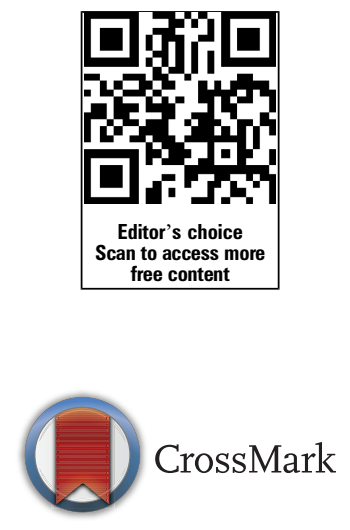

To cite: Bateman AC. J Clin Pathol 2014;67:865-874.

\begin{abstract}
The concept of serrated colorectal neoplasia has become recognised as a key process in the development of colorectal cancer (CRC) and an important alternative pathway to malignancy compared with the longestablished 'adenoma-carcinoma' sequence. Increasing recognition of the morphological spectrum of serrated lesions has occurred in parallel with elucidation of the distinct molecular genetic characteristics of progression from normal mucosa, via the 'serrated pathway', to CRC. Some of these lesions can be difficult to identify at colonoscopy. Challenges for pathologists include the requirement for accurate recognition of the forms of serrated lesions that are associated with a significant risk of malignant progression and therefore the need for widely disseminated reproducible criteria for their diagnosis. Alongside this process, pathologists and endoscopists need to formulate clear guidelines for the management of patients with these lesions, particularly with respect to the optimal follow-up intervals. This review provides practical guidance for the recognition of these lesions by pathologists, a discussion of 'serrated adenocarcinoma' and an insight into the distinct molecular genetic alterations that are seen in this spectrum of lesions in comparison to those that characterise the classic 'adenoma-carcinoma' sequence.
\end{abstract}

\section{INTRODUCTION}

Until relatively recently the only serrated ('saw tooth') colorectal lesion that many diagnostic histopathologists were aware of was the hyperplastic polyp. The term 'serrated polyp' was first used in 1990 by Longacre and Fernoglio-Preiser to describe a newly recognised form of colorectal polyp that showed features of a conventional adenoma and a hyperplastic polyp. This lesion subsequently became known as the 'traditional' serrated adenoma (TSA). ${ }^{1}$ Torlakovic and Snover later identified subtle differences between sporadically occurring hyperplastic polyps and the polyps found in the condition initially known as 'hyperplastic polyposis'. These polyps showed a constellation of features that were distinct from both sporadic hyperplastic polyps and TSAs, and this led to the recognition of the 'sessile serrated lesion' (SSL). ${ }^{2}$ SSLs can of course occur sporadically as well as in the setting of polyposis. Jass later demonstrated that SSLs were associated with a distinct molecular pathway to colorectal cancer (CRC). ${ }^{3}$ Jass highlighted the biological importance of these 'hyperplastic polyp-like' lesions that were more commonly found within the right colon, were usually sessile and relatively large (often $10 \mathrm{~mm}$ or more in diameter) but that did not show features of dysplasia as seen in 'classical' adenomas. ${ }^{4}$

As a result of these and other studies, a spectrum of colorectal polyps exhibiting a partially or wholly serrated architecture is now recognised (table 1). Some of these lesions show no dysplasia of any recognisable form while others show 'dysmaturation' that is now recognised by at least some pathologists as a subtle form of dysplasia. Finally, some serrated lesions show 'conventional' dysplasia, as is already widely recognised by histopathologists as an integral feature of 'classical' colorectal adenomas. These areas of 'conventional' dysplasia may be low grade or high grade in nature and in the setting of serrated colorectal polyps, and are usually present within one or more areas of the polyp, combined with other areas that do not show 'conventional' dysplasia. This heterogeneous appearance has led to use of the term 'mixed polyp' by some groups.

During the process of recognition of the serrated colorectal polyp 'spectrum', several names have been used to describe some of these lesions and this has led to terminological confusion. The key skill for the diagnostic histopathologist is the ability to recognise that some colorectal lesions that would probably previously have been called 'hyperplastic polyps', with the implication that they are not associated with a significant increase in CRC risk, may in fact represent one of the forms of colorectal polyp that can progress to malignancy.

The ability of histopathologists to differentiate accurately between types of serrated lesion is most pertinent during the differentiation between SSLs and hyperplastic polyps, as SSLs are the lesions that may not show conventional dysplasia, yet are associated with an increased risk of progression to CRC.

CRC arising in association with serrated polyps most often shows histological features that are not distinguishable from those of CRC arising in association with 'classical' adenomas. Alternatively, it may show a range of morphological appearances that are characteristic of 'serrated adenocarcinoma'. ${ }^{7}$ The molecular alterations occurring during progression to CRC along the 'serrated pathway' are distinct to those occurring within the classical 'adenoma-carcinoma sequence', and there is evidence that this progression occurs more quickly within the 'serrated pathway'.

\section{THE SPECTRUM OF SERRATED LESIONS Hyperplastic polyp}

Hyperplastic polyps are very commonly encountered by all pathologists who report colorectal lesions. They occur at all sites within the large intestine-although they are most common within the distal colon and rectum - and are classically less than $10 \mathrm{~mm}$ in size. They share some histological features with SSLs, for example, a serrated architecture. Three morphological variants exist-microvesicular, goblet cell and mucin-poor (figure 1). Very few pathologists will use this subclassification 
Table 1 Prevalence of the different types of serrated lesion within the colorectum (adapted from Bettington et al unless otherwise stated $)^{5}$

\begin{tabular}{ll}
\hline Lesion & Suggested prevalence \\
\hline Hyperplastic polyp & $25-30 \%$ of all colorectal polyps \\
& $10-20 \%$ of Western adults \\
Sessile serrated lesion (SSL) & $1.7-9 \%$ of all colorectal polyps \\
SSL with 'conventional' dysplasia & $13.2 \%$ of all SSLs \\
Traditional serrated adenoma & $0.6-1.9 \%$ of all colorectal polyps \\
Serrated adenocarcinoma & $9-12 \%$ of all colorectal adenocarcinomas \\
\hline
\end{tabular}

system for hyperplastic polyps in a routine setting. Despite this, knowledge of the spectrum of appearances of hyperplastic polyps may facilitate their positive identification and therefore facilitate their differentiation from other serrated lesions, especially SSLs (table 2).

Hyperplastic polyps have historically not been considered as precursor lesions to CRC. However, both BRAF and KRAS mutations (see later) are common in these lesions and are likely to be important steps in their development. ${ }^{8}$

\section{Sessile serrated lesions}

SSLs (also known as 'sessile serrated polyps' or 'sessile serrated adenomas') resemble hyperplastic polyps on initial examination. Indeed, differentiation between hyperplastic polyps and SSLs can be problematic, especially with small biopsies or those showing crush, diathermy or tangential cutting artefact. SSLs are most commonly encountered in the right colon, ${ }^{2}$ although they can occur throughout the large intestine. As their name suggests, they are usually sessile in nature (itself a difficult quality to define with absolute clarity). They may be over $10 \mathrm{~mm}$ in diameter, although interestingly, around one-third of SSLs are $5 \mathrm{~mm}$ or less across. ${ }^{2}$ The characteristic histological features of SSLs are listed in box 1 (figure 2). There is some difference of opinion regarding how many of these characteristic features are required and how widespread they need to be in order to make a diagnosis of SSL. For example, the American Gastroenterology Association Guidelines suggest that when assessing a serrated lesion, the presence of a single crypt
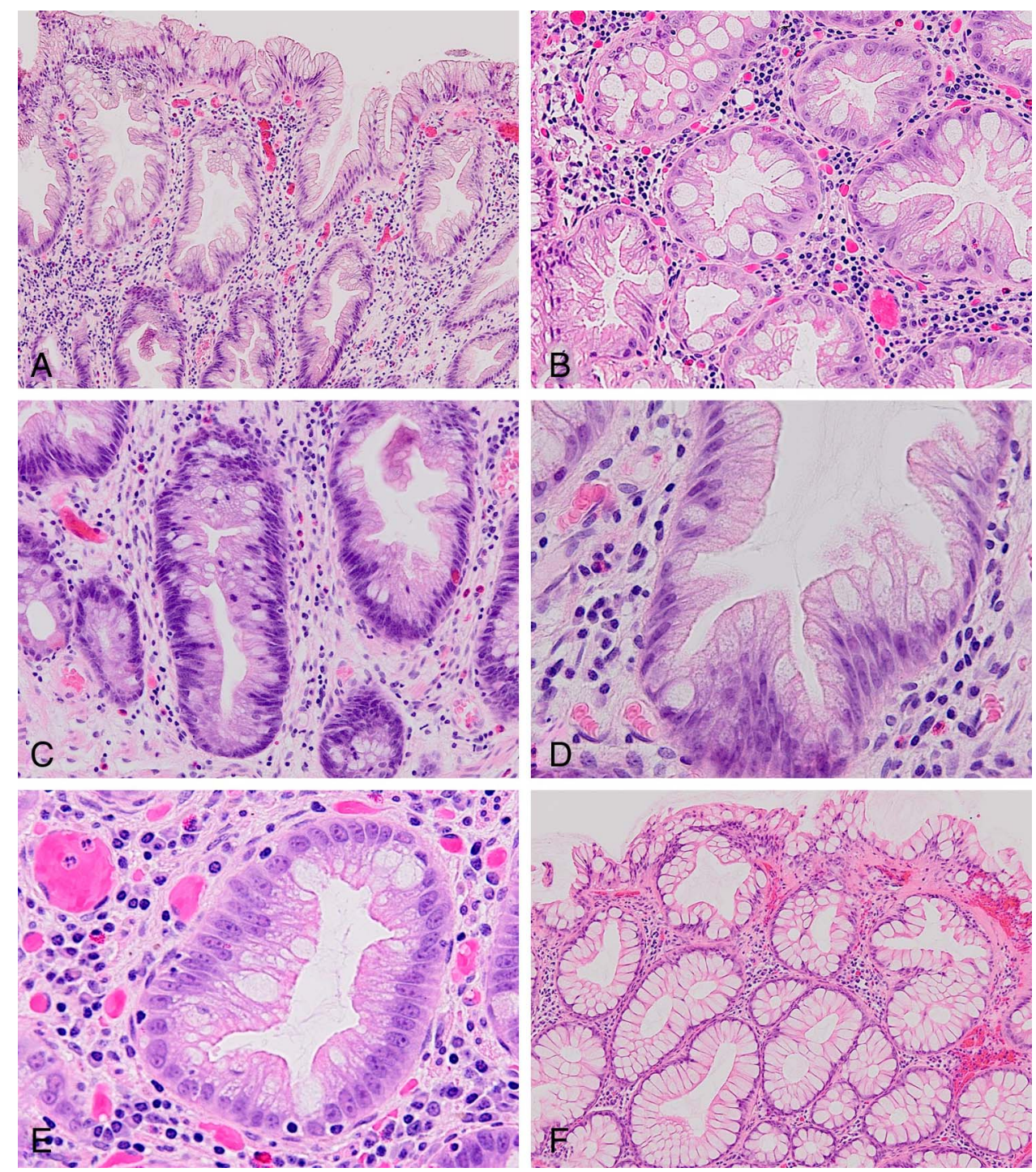

Figure 1 The histological features of hyperplastic polyps. (a) Microvesicular variant. Magnification $\times 100$. (b) Microvesicular variant. Magnification $\times 200$. (c) Base of crypts to show proliferative zone. Magnification $\times 200$. (d) Mucin-poor variant. Magnification $\times 400$. (e) Microvesicular variant. Magnification $\times 400$. (f) Goblet cell variant. Magnification $\times 100$. All-H\&E stain. 
Table 2 Key features of the three variants of hyperplastic polyp

\begin{tabular}{|c|c|c|}
\hline Variant & Clinical & Histological features \\
\hline Microvesicular & $\begin{array}{l}\text { Mainly found in the } \\
\text { distal colon and } \\
\text { rectum } \\
\text { Usually less than } \\
5 \mathrm{~mm} \text { diameter }\end{array}$ & $\begin{array}{l}\text { Well-organised arrangement of crypts } \\
\text { Serration and goblet cells limited to } \\
\text { superficial half of crypts } \\
\text { Microvesicular mucin droplets in } \\
\text { epithelial cells* } \\
\text { Thickened basement membrane } \\
\text { Vertically orientated smooth muscle } \\
\text { fibres }\end{array}$ \\
\hline Goblet cell & $\begin{array}{l}\text { Distal colon and } \\
\text { rectum } \\
\text { Almost always less } \\
\text { than } 5 \mathrm{~mm} \text { diameter }\end{array}$ & $\begin{array}{l}\text { May resemble normal mucosa on initial } \\
\text { examination } \\
\text { Mucosal thickening } \\
\text { Prominent mature goblet cells } \\
\text { Surface tufting }\end{array}$ \\
\hline Mucin poor & Rare & $\begin{array}{l}\text { Similar to microvesicular type but both } \\
\text { goblet cells and microvesicular mucin } \\
\text { are less evident }\end{array}$ \\
\hline
\end{tabular}

showing one of the characteristic features is sufficient in order to diagnose a SSL. ${ }^{9}$ In contrast, the World Health Organisation criteria include a statement that at least three crypts-or two adjacent crypts-must show the characteristic features for the diagnosis to be reached. ${ }^{10}$ SSLs share some histological features with the microvesicular variant of hyperplastic polyp, while $B R A F$ mutations are common within both lesions. These features have led some to suggest that SSLs may evolve from hyperplastic polyps. ${ }^{8}$ If this is true and hyperplastic polyps are indeed part of a spectrum of serrated polyps that includes SSLs, it clearly leads to the requirement for reassessment of the relationship (if any) of hyperplastic polyps to CRC.

\section{Box 1 Key histological features of SSLs ${ }^{2}$}

Irregular distribution of crypts

Dilatation of crypt bases

Serration present at crypt bases

Branched crypts

Horizontal extension of crypt bases*

Dysmaturation of crypts $\dagger$

Herniation of crypts through muscularis mucosa

World Health Organisation criteria-at least three crypts or at

least two adjacent crypts must show one or more of these

features to enable a diagnosis of SSL ${ }^{10}$

American Gastroenterology Association criteria-one crypt showing the characteristic features is sufficient for the diagnosis of $\mathrm{SSL}^{9}$

*Involved crypts often have an ' $L$ ' or inverted ' $T$ ' shape. tDysmaturation is disordered cellular maturation within crypts and is evidenced by subtle nuclear enlargement, crowding, pseudostratification and mitotic activity together with the presence of a disorganised mixture of non-mucus-containing epithelial cells and mature goblet cells within the deep aspects of crypts. ${ }^{2}$ In this context, assessment of proliferation index, for example, using MIB-1 may provide supporting evidence for a diagnosis of SSL by highlighting epithelial cell proliferation within the superficial half of crypts. However, such immunohistochemistry, while sometimes helpful, does not reveal features that are alone diagnostic of SSL.

SSL, sessile serrated lesion.
There is evidence that the reproducibility of diagnosis of SSLs is poor, that is, that significant inter-observer variability exists in the differentiation of these lesions from other polyps. ${ }^{11} \mathrm{~A}$ recent single-centre study has demonstrated a large increase in the diagnosis of SSLs over a 4-year period from 2009, but also that retrospective review of right-sided lesions originally diagnosed as hyperplastic polyps resulted in re-categorisation to SSLs in $30-64 \% .^{12}$ It has been suggested that the presence of features of mucosal prolapse may be one of the most frequent reasons for misdiagnosis of SSLs. ${ }^{13}$ Since SSLs appear to possess greater clinical significance than hyperplastic polyps and may progress more rapidly to adenocarcinoma than 'classical' adenomas, accurate diagnosis is essential. In the UK, the introduction of the National Bowel Cancer Screening Programme (BCSP) has raised the awareness of all forms of colorectal polyp, including SSLs. Educational events linked to the BCSP should gradually improve the future consistency of diagnosis of these lesions among pathologists involved in the programme. There is some evidence that achieving a consensus on the diagnostic criteria for serrated lesions (including hyperplastic polyps and TSAs) between reporting pathologists can improve the consistency of diagnosis of these lesions. ${ }^{14}$

'Pure' SSLs do not show 'conventional' dysplasia, that is, dysplasia as is characteristic of 'classical' adenomas, although they do characteristically show 'dysmaturation' (box 1). However, dysplasia can develop within them-both low and high grade. The natural history of SSLs with and without 'conventional' dysplasia is not fully defined. However, it is believed that the development of 'conventional' dysplasia is indicative of a high risk of progression to CRC and that malignancy may supervene more rapidly than with 'classical' adenomas. ${ }^{5} 15$ The term 'mixed polyps' has been used to describe SSLs that include an area of 'conventional' dysplasia. However, this term may not be ideal as it could be interpreted as implying that these lesions develop de novo as a combination of SSL and 'conventional' dysplasia rather than following the occurrence of 'conventional' dysplasia within a pre-existing SSL that originally developed without 'conventional' dysplasia. The alternative term 'sessile serrated adenoma' has been suggested to describe SSLs with (or sometimes, indeed, without) 'conventional' dysplasia. The use of this term is understandable as the areas of 'conventional' dysplasia in these lesions frequently possess a serrated morphology, even when it is high grade in nature. ${ }^{15}$ However, at present, the phrase 'sessile serrated adenoma' is used more commonly in North America than in the UK.

'Conventional' dysplasia is recognisable when it occurs in SSLs using the same criteria for its recognition in 'classical' adenomas. However, loss of the DNA mismatch repair enzymes hMLH-1 and hMSH-2 is commonly seen in 'conventional' dysplasia arising in SSLs, and therefore demonstration of loss of expression of these proteins using immunohistochemistry may be useful to confirm the presence of dysplasia occurring in this setting. ${ }^{16}$ However, since loss of DNA mismatch repair enzyme expression occurs in these lesions due to inactivation of the gene promoter sequence, demonstration of loss of expression of these proteins in this context does not imply that the patient has Lynch syndrome, that is, a germline mutation in the corresponding gene (figure 3 ) (see later).

\section{Traditional serrated adenomas}

TSAs are relatively uncommon lesions that occur most frequently in the left colon and are characterised by tubulovillous architecture, eosinophilic cytoplasm, elongated ('pencillate') nuclei and ectopic crypts, that is, the presence of multiple tiny crypts extending from the primary crypts (figure 4). The latter 

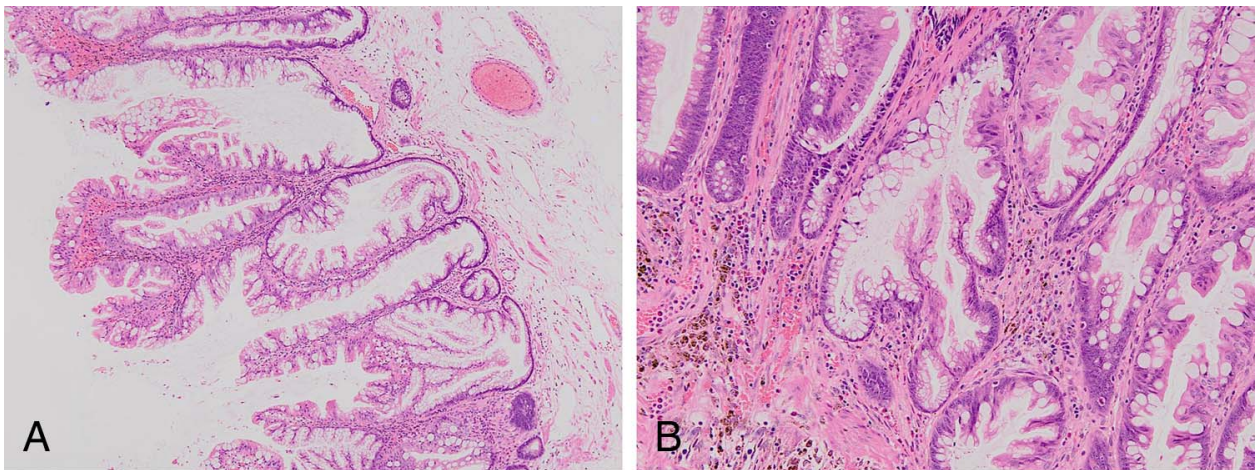

impart a serrated appearance to the lesion. These areas are usually mixed with areas showing a more typical adenoma growth pattern, and the transition between these areas is classically abrupt. High-grade dysplasia can occur within TSAs and progression to adenocarcinoma may take place, with the latter showing a serrated appearance (see below). ${ }^{7}$ Occasionally, TSAs may show a strikingly filiform growth pattern. ${ }^{17}$
The proportion of a lesion that is required to show the characteristic features of a TSA-in contrast to areas showing the appearances of a 'classical' adenoma-in order to make a diagnosis of TSA is not clearly defined. Neoplastic epithelium with a focally eosinophilic appearance may be seen in lesions that otherwise show the features of a 'classical' tubulovillous adenoma and without the constellation of features that are 

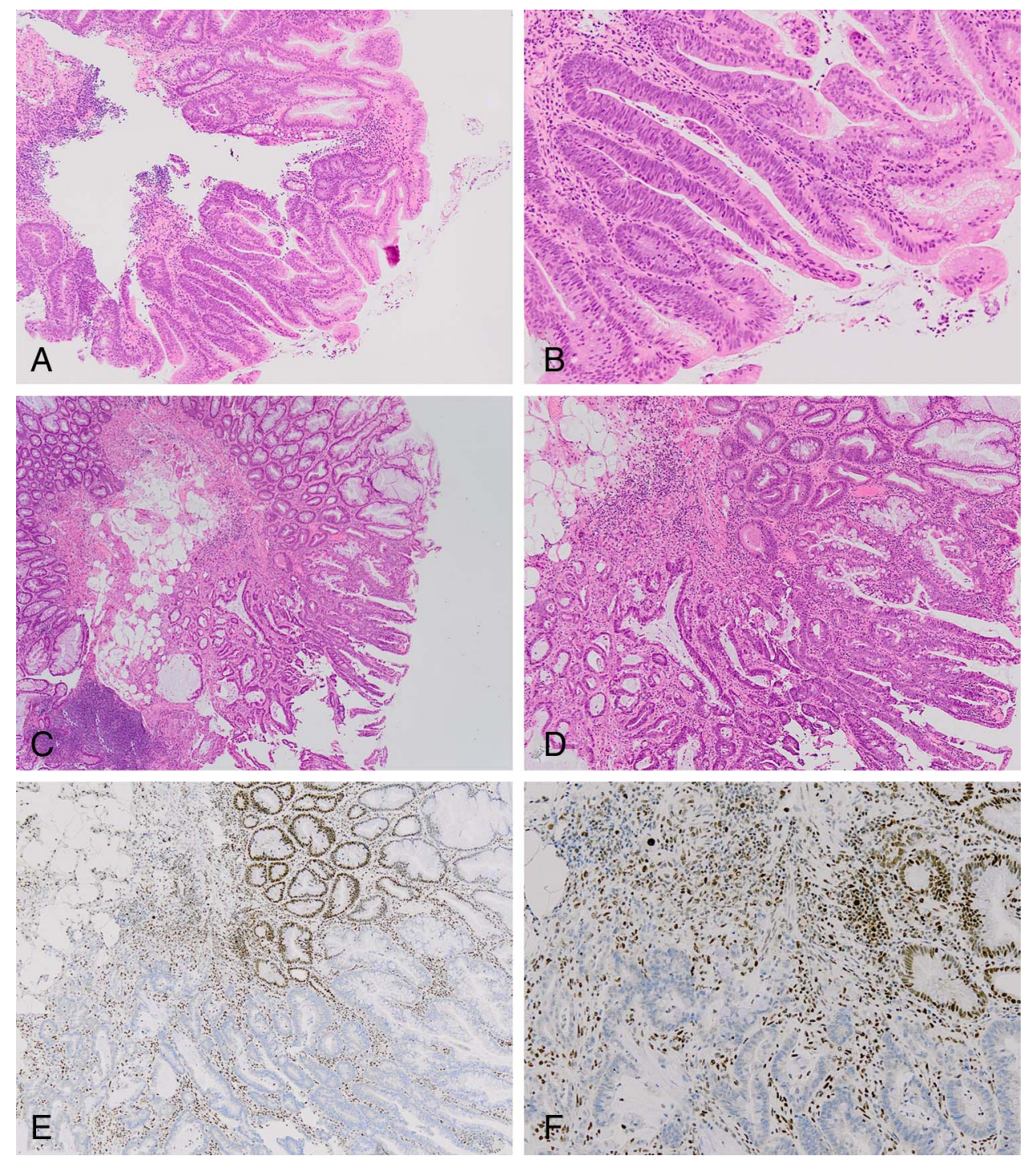

Figure 3 Sessile serrated lesion (SSLs) with 'conventional' dysplasia and adenocarcinoma. (a) Low-grade 'conventional' dysplasia arising within an SSL (lower half of field). Magnification $\times 40$. (b) As (a). Magnification $\times 100$. (c) 'Conventional' dysplasia and adenocarcinoma arising within an SSL (lower half of field). Magnification $\times 20$. (d) As (c). Magnification $\times 40$. (a) to (d)-all-H\&E stain. (e) Immunohistochemistry to show loss of nuclear hMLH-1 expression within area of dysplasia and adenocarcinoma. Magnification $\times 40$. (f) As (e). Magnification $\times 100$.

characteristic of TSAs (figure 4). These lesions are best regarded as 'classical' adenomas. However, some lesions comprise almost equal proportions of TSA-like and 'classical adenoma'-like areas, and it is likely that these will be termed TSAs by some and 'mixed' TSA and 'classical' adenomas by others. Regardless of the precise terminology used in this situation, the key step is to recognise the lesion as a variant of an adenoma and to be able to grade the dysplasia accurately, as these assessments will allow the correct risk stratification.

\section{Endoscopic recognition of serrated lesions}

SSLs are often difficult to recognise using conventional endoscopy (figure 5). Presumably this is due to their flat growth pattern and their not uncommon association with mucosal folds. They are also prone to being covered within mucus. ${ }^{18}$ Endoscopic identification rates for SSLs vary significantly between studies-from $1 \%$ to $18 \%$ in one study. ${ }^{19}$ Advanced techniques such as magnifying endoscopy and narrow band imaging may enhance their visualisation. ${ }^{20} 21$ The location and size of serrated lesions can help the endoscopist to determine whether they are likely to be dealing with an HP, an SSL or a TSA. TSAs are more commonly pedunculated, while some have suggested that they possess a red colouration on endoscopic examination-although this does not seem to be a universally held belief. ${ }^{18}$

\section{SERRATED POLYPOSIS}

Serrated polyposis (aka hyperplastic polyposis) is a condition characterised by the presence of multiple serrated polyps within the colorectum. Criteria for the diagnosis of this condition have now been created (box 2). ${ }^{10}$ The distinct morphological features of these polyps were described by Torlakovic and Snover in 1996, highlighting that the polyps in this syndrome showed important differences from those of sporadic hyperplastic polyps. ${ }^{2}$ The polyps found in serrated polyposis may be quite variable in histological appearance, even within the same patient. However, the majority show features most in keeping with those of SSLs, with some showing the appearances of 'classical' adenomas. ${ }^{2}$ The fact that the majority of lesions in patients with this condition show the features of SSLs explains why- 

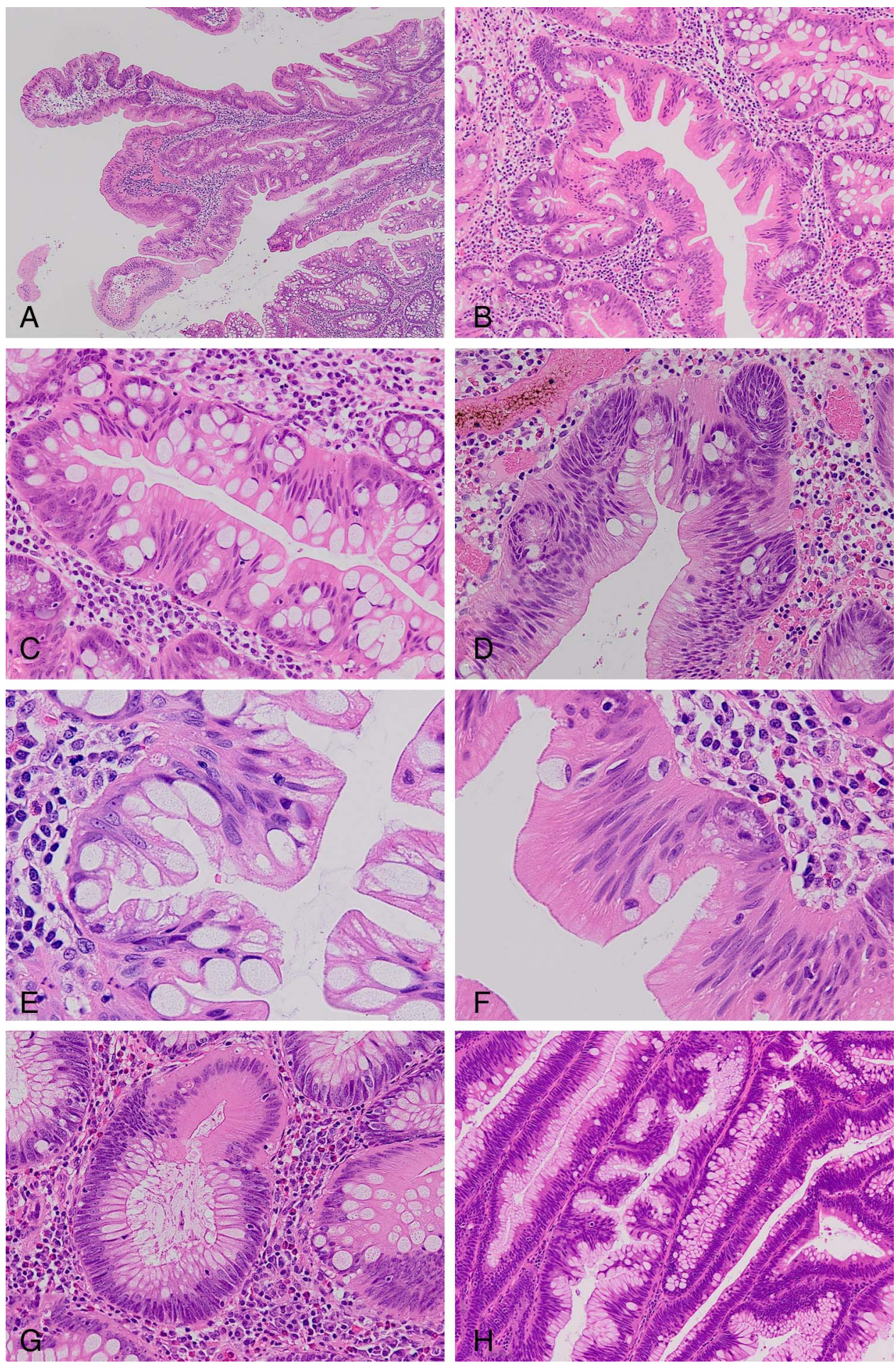

Figure 4 The histological features of traditional' serrated adenomas (TSAs). (a) Low-power view to show filiform architecture. Magnification $\times 40$. (b) Eosinophilic cytoplasm and pencillate nuclei with crypt budding. Magnification $\times 100$. (c) and (d) Crypt budding. Magnification $\times 200$. (e) Crypt budding. Magnification $\times 400$. ( $\mathrm{f})$ Eosinophilic cytoplasm and pencillate nuclei. Magnification $\times 400$. (g) A tiny focus of eosinophilic cytoplasm and pencillate nuclei within an otherwise typical 'classical' tubulovillous adenoma. Magnification $\times 200$. (h) A focus of crypt budding within an otherwise typical 'classical' tubulovillous adenoma. Magnification $\times 100$. All- H\&E stain.

prior to the identification of SSLs as a distinct entity-the condition was known as 'hyperplastic polyposis'.

\section{SERRATED ADENOCARCINOMA}

It is well established that serrated polyps can progress to CRC (box 3 ) and the term 'serrated adenocarcinoma' has been used to describe these tumours. ${ }^{7} 22$ Just as CRC arising within the classical 'adenoma-carcinoma' pathway may be associated with an adjacent residual adenomatous component, serrated polyps are sometimes visible at the edge of CRC arising within the 'serrated' pathway. Second, the invasive carcinoma may itself show morphological features that are characteristic of CRC arising in 


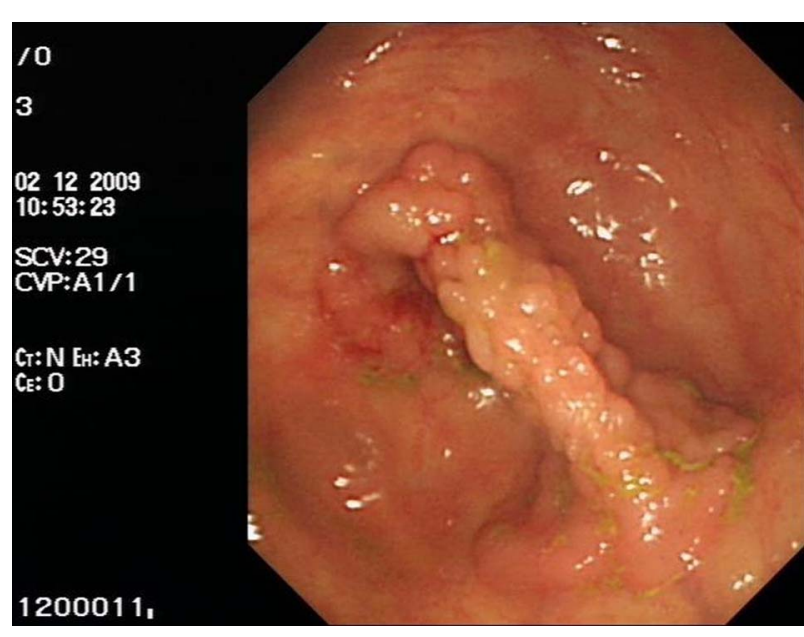

Figure 5 The endoscopic appearance of a sessile serrated lesion.

the context of the 'serrated' pathway. Before the link between serrated polyps and 'serrated adenocarcinoma' was established, the features of 'serrated adenocarcinoma' were described within cancers that were MSI-H but sporadic in nature, that is, not arising on the basis of Lynch syndrome. It is very likely that many, if not all, of these cancers in fact arise via the 'serrated' pathway. It is currently thought that around a third of cancers that arise from the 'serrated pathway' show a serrated morphology-therefore, the majority show features that are not distinguishable from cancers arising from the 'classical' adenoma-carcinoma sequence. ${ }^{5}$

The key histological features of 'serrated adenocarcinomas' that distinguish them from 'conventional' adenocarcinomas are listed in table 3. Of these appearances, the serrated growth pattern is the most common and the trabecular pattern is the rarest.

The majority of 'serrated adenocarcinomas' arise in the distal colon or rectum. These tumours are MSI-S or exhibit MSI-L and are believed to develop from TSAs. A minority arise within the caecum and ascending colon, show MSI-H and are thought to arise from SSLs. ${ }^{23}$

There is some evidence that serrated adenocarcinomas possess a worse prognosis than CRC developing along the 'adenomacarcinoma' pathway. In particular, they more commonly show adverse histological factors such as tumour budding. ${ }^{24}$ Some will also contain KRAS mutations, and these tumours will be resistant to anti-EGFR therapies in the same way to KRAS-mutant CRC arising from the 'adenoma-carcinoma' pathway (see below).

\section{Box 2 Criteria for the diagnosis of serrated polyposis ('hyperplastic polyposis') ${ }^{10}$}

At least five histologically diagnosed serrated polyps proximal to the sigmoid colon, of which two are $>10 \mathrm{~mm}$ in diameter Any number of serrated polyps occurring proximal to the sigmoid colon in an individual who has a first degree relative with serrated polyposis

More than 20 hyperplastic polyps of any size but distributed throughout the colon

\section{Box 3 Evidence supporting the link between sessile} serrated lesions and colorectal carcinoma

Progression to carcinoma of lesions originally thought to be hyperplastic polyps

Carcinoma developing in serrated (hyperplastic) polyposis

Presence of dysplasia (conventional and serrated type) in sessile serrated lesions

Metachronous carcinoma in patients with sessile serrated lesions

Presence of sessile serrated lesions adjacent to carcinomas

Morphological and immunohistochemical similarities of some

carcinomas to sessile serrated lesions

Mutations and epigenetic phenomena common to various

stages of the serrated neoplasia pathway

Similarities of carcinomas and sessile serrated lesions on DNA array analysis

\section{GENETIC ALTERATIONS IN SERRATED LESIONS AND PROGRESSION TO CANCER}

In parallel with the recognition of the morphological features of SSLs came an understanding of the distinct molecular genetic alterations that characterise these lesions. The genes that are most involved in the progression from normal mucosa to CRC are listed in table 4 . In addition, it is important to understand the concepts of microsatellite instability, the $\mathrm{CpG}$ island methylator phenotype (CIMP) and the Wnt signalling pathway.

The KRAS gene encodes the KRAS protein (a proto-oncogene), which is a member of the Ras family of proteins that are very important for signalling in normal cells. Mutations within the KRAS gene are commonly found in carcinomas of the pancreas, lung and colorectum and result in KRAS acting as an oncogene. In CRC, the presence of a KRAS mutation is also a predictor of a poor response to EGFR inhibitors such as cetuximab. This is because certain KRAS mutations result in the KRAS protein becoming self-activating and because KRAS is downstream of EGFR in the signal transduction pathway; pharmacological inhibition of EGFR does not then prevent (aberrant) signal transduction via this pathway when KRAS is mutant. ${ }^{25}$

Table 3 The key distinguishing features of serrated adenocarcinomas $^{23}$

\begin{tabular}{ll}
\hline Subtype & Detailed appearances \\
\hline Serrated & $\begin{array}{l}\text { Well-moderately differentiated glands in which the epithelium has a } \\
\text { serrated appearance } \\
\text { Eosinophilic cytoplasm-may be intense* } \\
\text { Vesicular (open chromatin) nuclei with basal location* } \\
\text { Dirty necrosis focal or absent } \\
\text { May possess small areas of mucinous differentiation }\end{array}$ \\
Mucinous & $\begin{array}{l}\text { The neoplastic cells are present usually as (poorly differentiated) } \\
\text { cords of cells floating within the mucus pools }\end{array}$ \\
& 'Serrated cytology' retained \\
& $\begin{array}{l}\text { Areas with a 'serrated morphology' can form up to } 50 \% \text { of the } \\
\text { tumour }\end{array}$ \\
Trabecular & $\begin{array}{l}\text { Poorly differentiated clusters and cords of neoplastic epithelial cells } \\
\text { 'Serrated cytology' maintained } \\
\text { Lymphatic invasion common }\end{array}$ \\
& $\begin{array}{l}\text { This pattern may be seen at the advancing edge of serrated } \\
\text { adenocarcinomas that are otherwise one of the other two subtypes }\end{array}$
\end{tabular}


Table 4 The genes most commonly involved (through inactivation, loss or mutation) during the molecular pathways from normal colorectal mucosa to CRC

\begin{tabular}{ll}
\hline Serrated pathways & Adenoma-carcinoma sequence* \\
\hline BRAF† & APC \\
KRAS & KRAS \\
$h M L H-1 \S$ & $p 53$ \\
$p 16$ & $S M A D 4$ \\
$M G M T$ & \\
\hline *Familial CRC proceeds down a very similar pathway to the adenoma-carcinoma \\
sequence; in familial adenomatous polyposis a germline mutation in the APC gene is \\
present while in Lynch syndrome a germline mutation in one of the DNA mismatch \\
repair enzymes is present. In both conditions, the second allele of the corresponding \\
gene is then lost or inactivated through mutation. \\
†Mutations in BRAF are an early event in the SSL pathway. \\
¥Mutations in KRAS are an early event in the TSA pathway. \\
ShMLH-1 is inactivated in the SSL pathway via the CIMP mechanism. \\
APC, adenomatous polyposis coli; CIMP, CpG island methylator phenotype; CRC, \\
colorectal cancer; SSL, sessile serrated lesion; TSA, traditional' serrated adenoma.
\end{tabular}

The BRAF gene (another proto-oncogene) encodes a protein called B-Raf, which is a member of the Raf kinase family of phosphorylating enzymes that are involved in the control of cell division and differentiation. Acquired BRAF mutations have been identified in many human cancers, including malignant melanoma and carcinomas of the lung and colorectum. These mutations result in $B R A F$ acting as an oncogene. Over 30 mutations have been recognised in the $B R A F$ gene, of which the V600E mutation is the most common $(90 \%$ of $B R A F$ mutations). The V600E mutation is a single-nucleotide substitution at codon 600 of the gene, leading to an amino acid change from valine $(\mathrm{V})$ to glutamate $(\mathrm{E})$ at this position. CRCs showing both loss of the hMLH-1 protein and the presence of the V600E mutation have lost hMLH-1 expression due to inactivation of the hMLH-1 encoding gene rather than due to an inherited mutation within the gene (ie, Lynch syndrome). Screening for the V600E mutation in this situation therefore reduces the number of cases in which a search for a mutation in the hMLH-1 encoding gene is required. ${ }^{26}$

The $\mathrm{p} 16$ gene is a tumour suppressor gene encoding a protein (cyclin-dependent kinase inhibitor 2A) that is involved in cell cycle control and that may be mutated in several different cancers. The MGMT gene encodes the MGMT protein (methylated DNA protein cysteine methyltransferase) that is involved in DNA repair and that can be inactivated via hypermethylation of its promoter sequence.

Microsatellites are short, non-coding regions of DNA that are scattered throughout the genome. They can act as markers of imperfect DNA replication since when this occurs individual microsatellites are present at differing length within different cells. This phenomenon is termed 'microsatellite instability' (MSI). MSI can be present only in some microsatellites (MSI-low or MSI-L) or within many (MSI-high or MSI-H). The presence of MSI-H is commonly associated with defective DNA mismatch repair (ie, the failure of the normal process of correction of imperfections in DNA replication) and is characteristic of Lynch syndrome and in certain CRCs that arise from the serrated pathway. Lesions that show no evidence of MSI are termed 'microsatellite stable' (MSS).

The CIMP is a state in which extensive methylation of the promoter sequences of genes-including those encoding certain DNA mismatch repair enzymes-occurs. CpG islands are pairs of cytosine and guanine nucleotides that are present mainly within the promoter regions of genes such as the DNA mismatch repair enzyme-encoding gene hMLH-1. When methylation (a physiological process important in the regulation of gene activity) of these $\mathrm{CpG}$ islands occurs, this results in inactivation of the corresponding gene. Methylation may be present at either a low (CIMP-L) or high (CIMP-H) level across the genome.

The Wnt signalling pathways are important cascades of proteins that are involved in signal transduction and control of cellular growth and differentiation. Genes encoding proteins within this pathway can act as oncogenes when their regulation becomes abnormal.

Several different molecular pathways to CRC exist (table 5). The classical Vogelstein 'adenoma-carcinoma' model has been established for many years and involves what is believed to be a stepwise accumulation in mutations within, for example, APC (adenomatous polyposis coli; commonly mutated early in CRC), p53 (an important tumour suppressor gene involved in halting cellular proliferation in the presence of DNA damage and promoting apoptosis if this damage cannot be repaired) and $S M A D-4$ genes (one of a family of genes encoding proteins involved in signal transduction within the TGF- $\beta$ pathway). Mutations in the KRAS gene may also occur, and the resulting CRCs are MSS and either CIMP-negative or CIMP-L.

Familial adenomatous polyposis (FAP) is associated with a germline mutation in the APC gene, and the resulting CRC are CIMP-negative and MSS. Lynch syndrome is associated with a germline mutation in one of the DNA mismatch repair enzyme-encoding genes, and the resulting CRC are CIMP-negative and show MSI (the latter is associated with resistance to 5-fluorouracil chemotherapy).

SSLs are associated with early BRAF mutations followed in some cases by loss of hMLH-1 expression due to hypermethylation of the promoter sequence leading to inactivation of the encoding gene, rather than due to a mutation. One study of 148 colorectal polyps found that $90 \%$ of SSLs contain the BRAF V600E mutation compared with $29 \%$ of (microvesicular) hyperplastic polyps, $36 \%$ of TSAs and 5\% of 'classical' adenomas. ${ }^{27}$ The resulting CRC contain BRAF mutations, are CIMP-H and exhibit MSI. SSLs in which hMLH-1 expression is not lost may alternatively show p16 and MGMT loss. The resulting CRC again contain BRAF mutations and exhibit CIMP-H but are MSS.

TSAs are particularly associated with early KRAS mutations and Wnt abnormalities. The resulting CRC contain KRAS mutations and exhibit CIMP-L but are MSS.

\section{MANAGEMENT OF SERRATED LESIONS}

Whether or not serrated polyps are identified within the context of the BCSP, the most appropriate management of affected

Table 5 Molecular classification of colorectal cancer ${ }^{3}$

\begin{tabular}{|c|c|c|c|c|c|}
\hline Type & CIMP & MSI & BRAF & KRAS & Pathway \\
\hline 1 & High & High & Mutation & WT & Serrated \\
\hline 2 & High & $\begin{array}{l}\text { Low or } \\
\text { stable }\end{array}$ & Mutation & WT & Serrated \\
\hline 3 & Low & $\begin{array}{l}\text { Low or } \\
\text { stable }\end{array}$ & WT & Mutation & $\begin{array}{l}\text { Serrated or } \\
\text { adenoma-carcinoma }\end{array}$ \\
\hline 4 & Neg & Stable & WT & WT & Adenoma-carcinoma \\
\hline 5 & Neg & High & WT & WT & $\begin{array}{l}\text { Adenoma-carcinoma } \\
\text { Lynch syndrome }\end{array}$ \\
\hline
\end{tabular}

Note that CIMP-H status may or may not result in MSI depending on whether $h M L H-1$ is one of the genes inactivated as a result of hypermethylation of its promoter sequence.

CIMP, CpG island methylator phenotype; MSI, microsatellite instability; WT, wild type (ie, not mutant). 
Box 4 A suggested colonoscopic surveillance protocol for SSLs ${ }^{28}$

The numbers of SSLs and 'classical' adenomas should be additive. For example, the finding of two classical adenomas $<10 \mathrm{~mm}$ and one SSL $<10 \mathrm{~mm}$ should lead to 3-yearly surveillance Distal small hyperplastic polyps are not associated with an increased risk of colorectal cancer and surveillance should not be increased in frequency because of these lesions unless $>20$ are present

If the pathologist is unable to distinguish between a hyperplastic polyp and an SSL for technical reasons (eg, tiny biopsy or tangential cutting), then all proximal serrated polyps should be considered to represent SSLS Patients with serrated polyposis should undergo 2-yearly surveillance after all lesions $>5 \mathrm{~mm}$ have been resected Patients undergoing piecemeal resection of an SSL should undergo a site check at 2-6 months

If a large proximal SSL or multiple SSLs are identified, the use of advanced endoscopic techniques should be considered as a means of increasing the detection rate of serrated polyposis SSL, sessile serrated lesion.

patients requires clarification. Despite the morphological and molecular genetic similarities between microvesicular hyperplastic polyps and SSLs, it is still widely believed that patients in whom hyperplastic polyps are identified do not require follow-up based on this finding alone. On the other hand, TSAs should already be universally recognised as a form of adenoma and patients with these lesions will be managed in the same way as those with 'classical' adenomas that exhibit otherwise similar characteristics. For example, within the BCSP this will depend on their number, size and grade of dysplasia.

The optimal management of patients harbouring SSLs is still under discussion. Demonstration of the link between SSLs and CRC has led to an understanding that SSLs-even those without 'conventional' dysplasia-are at least as significant as 'classical' adenomas in defining cancer risk. This concern has been heightened with realisation that the 'serrated pathway' to CRC may be more rapid than the classical 'adenoma-carcinoma' sequence (box 4). ${ }^{28}$ The development of UK guidelines for the management of SSLs is currently under discussion by the British Society of Gastroenterology. It is likely that guidance will advocate complete excision of SSLs, as well as clinical follow-up that is at least as frequent as for 'classical' adenomas. Patients in whom serrated polyposis is identified will require even more careful scrutiny.

\section{FUTURE ADVANCES}

Further elucidation of the molecular links between the entire spectrum of serrated lesions and CRC will help to inform the guidelines for patient management and follow-up. In particular, a better understanding of the speed of progression along the 'serrated pathway' to CRC may allow enhanced risk stratification for patients in whom these lesions are identified. The similarities between microvesicular hyperplastic polyps and SSLs require further detailed study, and this could lead to a change to the paradigm that sporadically occurring hyperplastic polyps possess no link with the future development of CRC.

\section{Key messages}

- 'Serrated neoplasia' refers to a range of colorectal lesions with varying degrees of malignant risk, together with distinct forms of adenocarcinoma.

- Bowel Cancer Screening Programmes have highlighted to histopathologists the importance of recognizing and understanding the biological significance of the spectrum of serrated lesions.

- The optimal terminology, minimum diagnostic criteria and most appropriate management strategies for some serrated lesions (especially the sessile serrated lesion) are still in evolution.

\section{Competing interests None.}

Provenance and peer review Not commissioned; internally peer reviewed.

\section{REFERENCES}

1 Longacre TA, Fenoglio-Preiser CM. Mixed hyperplastic adenomatous polyps/serrated adenomas: a distinct form of colorectal neoplasia. Am J Surg Pathol 1990;14:524-37

2 Torlakovic E, Snover DC. Serrated adenomatous polyposis in humans. Gastroenterology 1996;110:748-55.

3 Jass JR. Classification of colorectal cancer based on correlation of clinical, morphological and molecular features. Histopathology 2007;50:113-30.

4 Hiuchi T, Jass JR. My approach to serrated polyps of the colorectum. J Clin Pathol 2004;57:682-6.

5 Bettington $\mathrm{M}$, Walker N, Clouston A, et al. The serrated pathway to colorectal carcinoma: current concepts and challenges. Histopathology 2013;62:367-86.

6 Lash RH, Genta RM, Schuler CM. Sessile serrated adenomas: prevalence of dysplasia and carcinoma in 2139 patients. J Clin Pathol 2010;63:681-6.

7 Makinen MJ. Colorectal serrated adenocarcinoma. Histopathology 2007;50:131-50.

8 Yang S, Farraye YA, Mack C, et al. BRAF and KRAS mutations in hyperplastic polyps and serrated adenomas of the colorectum: relationship to histology and CpG island methylation status. Am J Surg Pathol 2004;28:1452-9.

9 Rex DK, Ahnen DJ, Baron JA, et al. Serrated lesions of the colorectum: review and recommendations from an expert panel. Am J Gastroenterol 2012;107:1315-29.

10 Snover DC, Ahnen DJ, Burt RW, et al. Serrated polyps of the colon and rectum and serrated polyposis. In: Bosman FT, Carneiro F, Hruban RH, Theise ND. eds. World Health Organisation Classification of Tumours of the Digestive System. 4th edn. Lyon: IARC Press, 2010:160.

11 Wong NACS, Hunt LP, Novelli MR, et al. Observer agreement in the diagnosis of serrated polyps of the large bowel. Histopathology 2009;55:63-6.

12 Gill P, Wang LM, Bailey A, et al. Reporting trends of right-sided hyperplastic and sessile serrated adenomas in a large teaching hospital over a 4-year period (20092012). J Clin Pathol 2013;66:655-8.

13 Huang CC, Frankel WL, Doukides T, et al. Prolapse-related changes are a confounding factor in misdiagnosis of sessile serrated adenomas in the rectum. Hum Pathol 2013:44:480-6.

14 Ensari A, Bilezikci B, Carneiro F, et al. Serrated polyps of the colon: how reproducible is their classification? Virchows Arch 2012;461:495-504.

15 Lazarus $\mathrm{R}$, Junttila $\mathrm{OE}$, Karttunen TJ, et al. The risk of metachronous neoplasia in patients with serrated adenoma. Am J Clin Pathol 2005;123:349-59.

16 Oh K, Redston M, Odze RD. Support for hMLH1 and MGMT silencing as a mechanism of tumorigenesis in the hyperplastic-adenoma-carcinoma (serrated) carcinogenic pathway in the colon. Hum Pathol 2005;36:101-11.

17 Yantiss RK, Oh KY, Chen YT, et al. Filiform serrated adenomas: a clinicopathologic and immunophenotypic study of 18 cases. Am J Surg Pathol 2007;31:1238-45.

18 Yamada A, Notohara K, Aoyama I, et al. Endoscopic features of sessile serrated adenoma and other serrated colorectal polyps. Hepatogastroenterology 2011;58:45-51

19 Kahi CJ, Li X, Eckert GJ, et al. High colonoscopic prevalence of proximal colon serrated polyps in average-risk men and women. Gastrointest Endosc 2012;75:515-20.

20 Ishigooka S, Nomoto M, Obinata N, et al. Evaluation of magnifying colonoscopy in the diagnosis of serrated polyps. World J Gastroenterol 2012;18:4308-16.

21 Hazewinkel Y, Lopez-Ceron M, East JE, et al. Endoscopic features of sessile serrated adenomas: validation by international experts using high-resolution white-light endoscopy and narrow-band imaging. Gastrointest Endosc 2013;77:916-24. 
22 Jass JR, Smith M. Sialic acid and epithelial differentiation in colorectal polyps and cancer-a morphological, mucin and lectin histochemical study. Pathology $1992 ; 24: 233-42$

23 Tuppurainen K, Makinen JM, Junttila O, et al. Morphology and microsatellite instability in sporadic serrated and non-serrated colorectal cancer. J Pathol 2005;207:285-94.

24 Garcia-Solano J, Conesa-Zamora P, Trujillo-Santos J, et al. Tumour budding and other prognostic pathological features at invasive margins in serrated colorectal adenocarcinoma: a comparative study with conventional carcinoma. Histopathology 2011;59:1046-56.
25 Shackelford RE, Whitling NA, McNab P, et al. KRAS testing: a tool for the implementation of personalized medicine. Genes Cancer 2012;3:7-8.

26 Pakneshan S, Salajegheh A, Smith RA, et al. Clinicopathological relevance of BRAF mutations in human cancer. Pathology 2013;45:346-56.

27 Carr NJ, Mahajan H, Tan KL, et al. Serrated and non-serrated polyps of the colorectum: their prevalence in an unselected case series and correlation of $B R A F$ mutation analysis with the diagnosis of sessile serrated adenoma. J Clin Pathol 2009;62:516-18.

28 Leedham S, East JE, Chetty R. Diagnosis of sessile serrated polyps/adenomas: what does this mean for the pathologist, gastroenterologist and patient? I Clin Pathol 2013;66:265-8. 